\title{
Editorial
}

\section{Joint Topics on Exotic Hadron States and Heavy Flavor Hadronic Decay}

\author{
Xian-Wei Kang $\mathbb{D}^{1},{ }^{1}$ J. A. Oller, ${ }^{2}$ Ling-Yun Dai, ${ }^{3}$ and Tao Luo $\mathbb{D}^{4}$ \\ ${ }^{1}$ College of Nuclear Science and Technology, Beijing Normal University, Beijing 100875, China \\ ${ }^{2}$ Departamento de Física, Universidad de Murcia, 30071 Murcia, Spain \\ ${ }^{3}$ School of Physics and Electronics, Hunan University, Changsha 410082, China \\ ${ }^{4}$ Key Laboratory of Nuclear Physics and Ion-Beam Application (MOE) and Institute of Modern Physics, Fudan University, \\ Shanghai 200443, China
}

Correspondence should be addressed to Xian-Wei Kang; kxw198710@126.com and Tao Luo; luot@fudan.edu.cn

Received 28 May 2019; Accepted 28 May 2019; Published 16 July 2019

Copyright (C) 2019 Xian-Wei Kang et al. This is an open access article distributed under the Creative Commons Attribution License, which permits unrestricted use, distribution, and reproduction in any medium, provided the original work is properly cited.

The internal structure of a hadron, as a key topic in the nuclear and particle physics, can be accessed by hadronic scattering, production, and/or its decay. As it is known Quantum Chromodynamics (QCD) is a nonperturbative quantum field theory at the low-energy region, and many approaches have been proposed for studying different aspects of this rich dynamics, e.g., the various quark models, BetheSalpeter (BS) equation approach, QCD sum rules, and lowenergy effective field theories. Flavor physics is a research field which allows us to probe the charm and beauty quark decays. On the other hand, the exotic heavy-quark hadron physics becomes very fashionable since the discovery of the $\mathrm{X}(3872)$. As we know QCD does not exclude the existence of exotic hadrons (with quantum numbers that do not fit for $q \bar{q}$ in the case of mesons and $99 q$ for baryons), but we still have no way to predict all the exotic hadrons from first-principle calculations (despite the advances in lattice QCD). Studies on the $X Y Z$ states certainly help our understanding on the underlying dynamics of QCD. Based on such circumstance, we propose a current special issue, where both theoretical and experimental papers have been attracted.

In the paper by F. Yan and B. Zheng, a tag method is proposed that allows a direct absolute measurement of the decay branching fractions of the $J / \psi$ with the data set at the $\psi(3686)$; namely, the $J / \psi$ is produced from the decay $\psi(3686) \longrightarrow J / \psi \pi^{+} \pi^{-}$. The next paper by P. G. Ortega et al. develops the unquenching of the quark model, where the quark-antiquark pair creation (or continuum coupling effect) is incorporated, and especially, the coupling between quark-antiquark states and meson-meson channels is treated nonperturbatively, an improvement compared to the Cornell Model. In the paper by J. Zhang et al., they make a combined fit to the measured cross sections of $e^{+} e^{-} \longrightarrow$ $\omega \chi_{c 0}, \pi^{+} \pi^{-} h_{c}, \pi^{+} \pi^{-} J / \psi, \pi^{+} \pi^{-} \psi(3686)$ and $\pi^{+} D^{0} D^{*-}+$ c.c., with the aim of better understanding the nature of the $Y(4220), Y(4390)$, and $Y(4660)$. They find that the two resonances $Y(4220)$ and $Y(4390)$ are sufficient to explain these cross sections below $4.6 \mathrm{GeV}$, and also the lower limits of their leptonic decay widths are determined. $\mathrm{H}$. Mutuk in the following paper revisits the potential model by using either the power potential or the logarithmic potential and obtains many physical quantities, including the spin averaged masses, hyperfine splittings, Regge trajectories of pseudoscalar and vector mesons, decay constants, leptonic decay widths, two-photon and two-gluon decay widths, and some allowed M1 transitions. He finds a good agreement with experimental data and other theoretical studies. In another paper by the same author, the mass spectra and decay constants of pseudoscalar and vector heavy-light mesons $\left(B, B_{s}, D, D_{s}\right)$ are investigated by applying QCD sum rules with a simple interpolating current, as well as within the quark model using the harmonic oscillator wave functions. Again, a good agreement with experiment is achieved. In the paper by Z.-Y. Wang et al., the authors conclude that the $X(5568)$ cannot be a $B \bar{K}$ molecular state by applying a method based on the BS equation, which is in line with 
many other theoretical points of view. More specifically, the BS equation is solved numerically in the covariant instantaneous approximation, with the interaction potential containing one-particle exchange diagrams and introducing two different form factors (monopole and dipole form factor) in the vertices.

In the next paper $\mathrm{H}$. Zhou et al. study the possibly enhanced $\mathrm{CP}$ violation in the singly Cabibbo-suppressed decay $D^{0} \longrightarrow K^{+} K^{-} \pi^{0}$. The authors consider the interference between the two intermediate resonances $K^{*}(892)^{+}$and $K^{*}(892)^{-}$and the amplitude is calculated in the factorizationassisted topological approach. Results show that the regional $\mathrm{CP}$ asymmetry in the overlapped region of the phase space can be an order of magnitude as large as $10^{-3}$, which may be accessed in the future high-statistic charm factories. In the paper by Y.-H. Chen, the author calculates the chromopolarizability of a quarkonium, which is a quantity describing the interaction between the quarkonium and soft gluonic fields. In the transition $\psi(3686) \longrightarrow J / \psi \pi \pi$, the nonperturbative $\pi \pi$ final state interaction (FSI) is treated model-independently with the use of the dispersion theory. The values of chromopolarizability are obtained, and it turns out that the FSI plays an indispensable role. In the paper by Z.-Y. Di and Z.-G. Wang, the $D \bar{D}^{*} K$ system is studied by employing QCD sum rules, and a resonance with mass $4.71_{-0.11}^{+0.19} \mathrm{GeV}$ is predicted. The future measurement of invariant mass of $J / \psi \pi K$ in the decay channel $B \longrightarrow J / \psi \pi \pi K$ can test such prediction. Finally, R. Gao et al. study the nature of the resonances $Z_{c}(3900), X(4020), \chi_{c 1}(4140), \psi(4260)$, and $\psi(4660)$ every of which lies nearby a threshold of two open heavy-flavor hadrons. The effective range expansion is used and the scattering length $(a)$ and effective range $(r)$ are determined by reproducing the pole positions. The composite or elementary nature of these states is addressed by considering the values of $a$ and $r$, as well as by calculating the compositeness coefficient $X$. Other interpretations of the latter coefficient in the literature are compared and critically reviewed.

\title{
Conflicts of Interest
}

We declare that we have no conflicts of interest.

\author{
Xian-Wei Kang \\ J. A. Oller \\ Ling-Yun Dai \\ Tao Luo
}



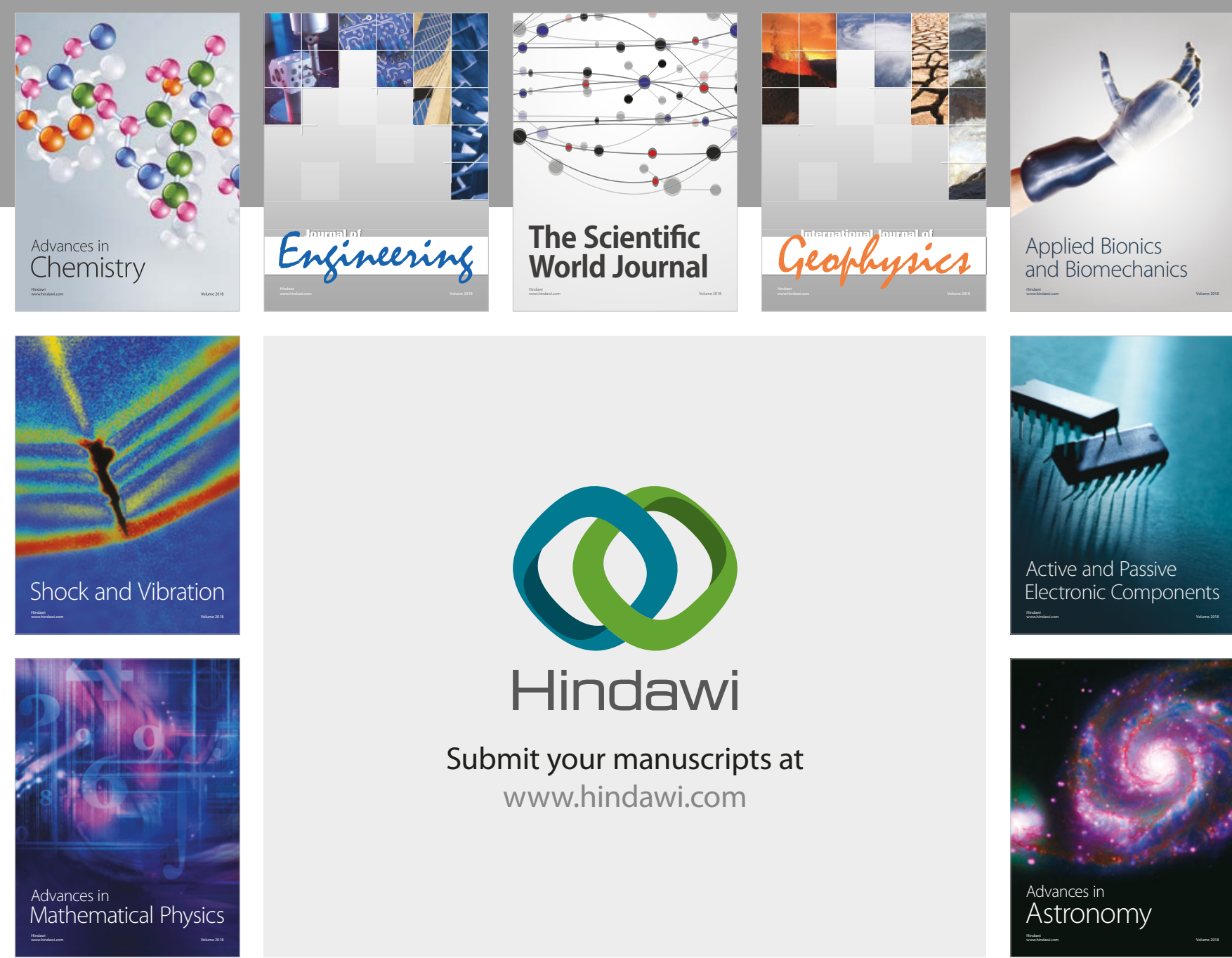

Submit your manuscripts at

www.hindawi.com

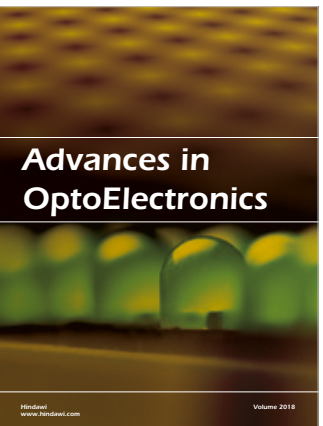

\section{Rotcting Machinery}
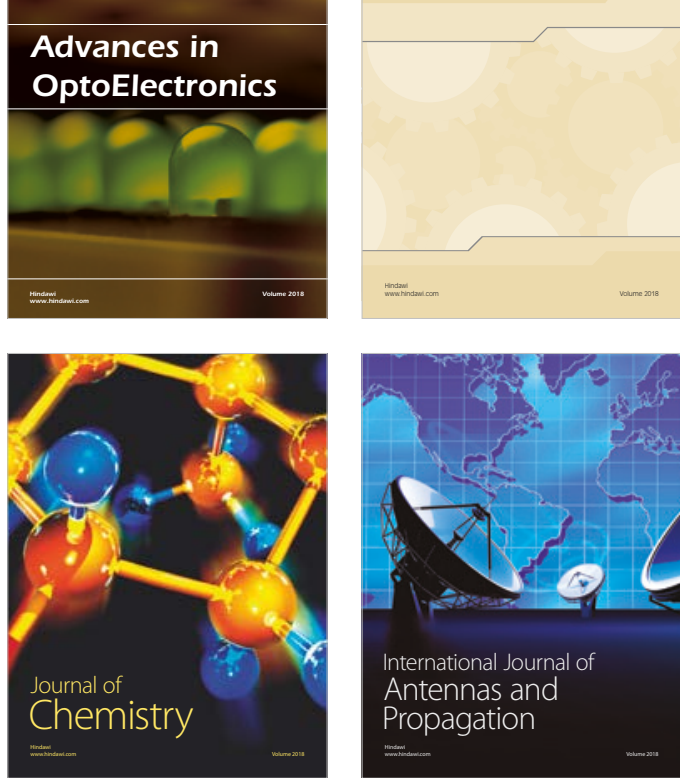

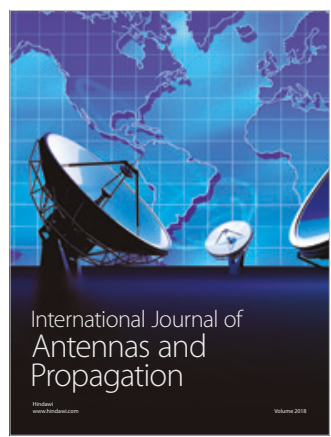

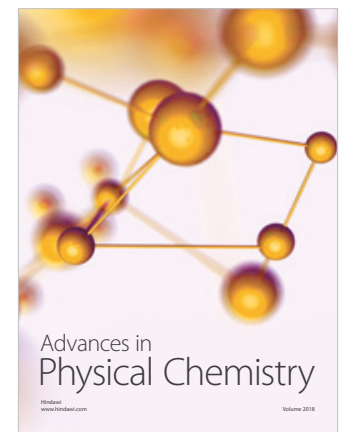

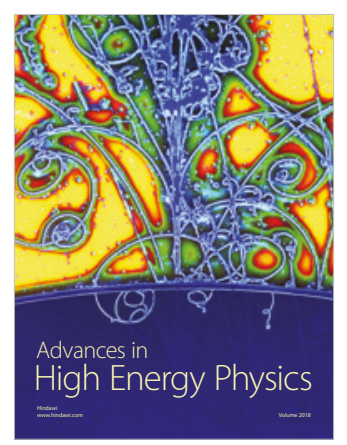

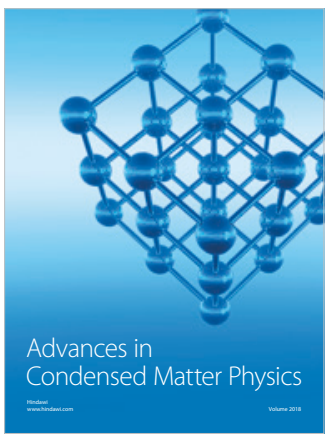

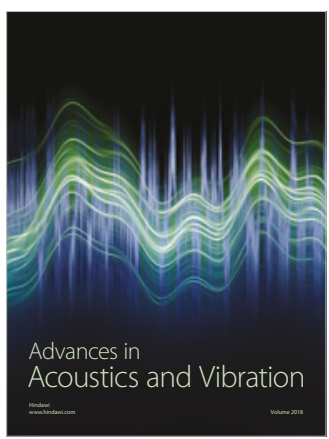

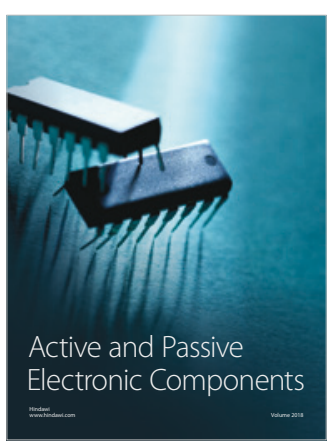
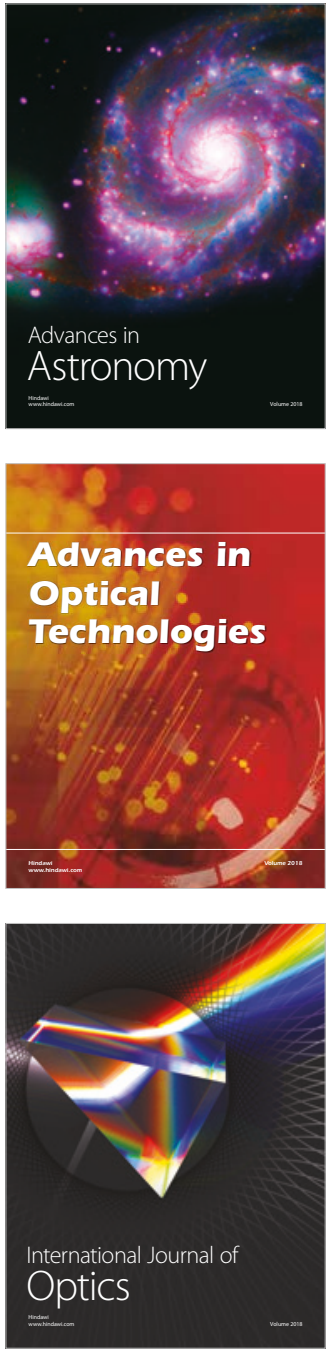